\title{
Critical examination of current response shift methods and proposal for advancing new methods
}

\author{
Véronique Sébille ${ }^{1}$ (D) Lisa M. Lix ${ }^{2}$. Olawale F. Ayilara ${ }^{2} \cdot$ Tolulope T. Sajobi $^{3} \cdot$ A. Cecile J. W. Janssens ${ }^{4}$. \\ Richard Sawatzky ${ }^{5}$ Mirjam A. G. Sprangers ${ }^{6}$ Mathilde G. E. Verdam ${ }^{6,7}$ - the Response Shift - in Sync Working Group
}

Accepted: 30 December 2020 / Published online: 17 February 2021

(c) The Author(s) 2021

\begin{abstract}
Purpose This work is part of an international, interdisciplinary initiative to synthesize research on response shift in results of patient-reported outcome measures. The objective is to critically examine current response shift methods. We additionally propose advancing new methods that address the limitations of extant methods.

Methods Based on literature reviews, this critical examination comprises design-based, qualitative, individualized, and preference-based methods, latent variable models, and other statistical methods. We critically appraised their definition, operationalization, the type of response shift they can detect, whether they can adjust for and explain response shift, their assumptions, and alternative explanations. Overall limitations requiring new methods were identified.

Results We examined 11 methods that aim to operationalize response shift, by assessing change in the meaning of one's self-evaluation. Six of these methods distinguish between change in observed measurements (observed change) and change in the construct that was intended to be measured (target change). The methods use either (sub)group-based or individual-level analysis, or a combination. All methods have underlying assumptions to be met and alternative explanations for the inferred response shift effects. We highlighted the need to address the interpretation of the results as response shift and proposed advancing new methods handling individual variation in change over time and multiple time points.

Conclusion No single response shift method is optimal; each method has strengths and limitations. Additionally, extra steps need to be taken to correctly interpret the results. Advancing new methods and conducting computer simulation studies that compare methods are recommended to move response shift research forward.
\end{abstract}

Keywords Patient-reported outcomes $\cdot$ Response shift $\cdot$ Operationalization $\cdot$ Methods

\section{Introduction}

Patient-reported outcome measures (PROMs) are incorporated into clinical practice and research to assess the impact of disease and treatment from the patient's viewpoint. PROMs may assess a range of outcomes, including

Véronique Sébille

veronique.sebille@univ-nantes.fr

1 UMR INSERM 1246, SPHERE “methodS in Patient-Centered Outcomes and HEalth ResEarch", University of Nantes, University of Tours, Nantes, France

2 Department of Community Health Sciences, University of Manitoba, Winnipeg, Canada

3 Department of Community Health Sciences and O'Brien Institute for Public Health, University of Calgary, Calgary, $\mathrm{AB}$, Canada for example quality of life, satisfaction with care, treatment preferences, and illness perceptions. Assessing change in PROM scores is crucial for monitoring patients' health status and to evaluate treatment effectiveness. However, PROM change scores may be invalidated by the occurrence of response shift. Response shift is particularly relevant for

4 Department of Epidemiology, Rollins School of Public Health, Emory University, Atlanta, USA

5 School of Nursing, Trinity Western University, Langley, BC, Canada

6 Department of Medical Psychology, Amsterdam University Medical Centers, Location AMC, Amsterdam Public Health Research Institute, Amsterdam, The Netherlands

7 Department of Methodology and Statistics, Institute of Psychology, Leiden University, Leiden, The Netherlands 
self-evaluation PROMs; these are PROMs that "yield measures rating experience as positive or negative compared with an internal standard" [1].

A range of response shift definitions have been proposed. In the context of organization research, Golembiewski et al. [2] proposed a typology of change including beta change (recalibration) and gamma change (reconceptualization). In parallel, the term 'response shift' was proposed by Howard et al. [3] in education research to explain the discrepancy between quantitative self-reports and interviews. They defined response shift as a change in internal standards or recalibration. Sprangers and Schwartz [4] subsequently introduced a working definition that combined and expanded the definitions proposed by Golembiewski et al. [2] and Howard et al. [3]. Specifically, they defined response shift as a change in the meaning of one's self-evaluation of a target construct that results from a change in one's internal standards (recalibration), a change in one's values (reprioritization), or one's redefinition of the target construct (reconceptualization). Subsequently, Rapkin and Schwartz [5] defined response shift as a change in appraisal that can explain an unexpected change in quality of life. Finally, Oort et al. [6, 7] highlighted that when the meaning of selfevaluation changes (the working definition of response shift [4]), the relationship between change in (observed) measurement (i.e., change in PROM scores) and change in the target construct (i.e., change in the construct that the PROM intends to measure) also changes. Hence, if response shift occurs, there is a discrepancy between observed change in PROM scores and change in the target construct. If no such discrepancy exists, then response shift cannot logically have occurred. However, it should be noted that a discrepancy between observed change and change in the target construct is a necessary but not a sufficient condition for response shift to occur. If alternative explanations can be ruled out, then the discrepancy between observed change and change in the target construct can be attributed to response shift (see also Vanier et al. [8]).

Response shift research calls for carefully designed studies $[9,10]$ and sound methods. A variety of response shift detection methods have been developed $[11,12]$ and applied in various clinical settings and populations [4, 13-18] Since some of these methods not only detect but also adjust for and explain detected response shift, we prefer to adopt the more general term of response shift methods in this paper. Some studies compared different methods to assess their convergent validity [19-23]. Methods have also been compared using simulated data [24]. All methods aim to detect response shift, but differ with respect to how they operationalize response shift (i.e., how response shift is evidenced in the results), their underlying assumptions, and alternative explanations for the observed response shift effects. These different methods call the interpretation of the results in terms of response shift into question. Indeed, one may wonder to what extent we can conclude that response shift has occurred if "response-shift effects" have been inferred by different methods using different operationalizations. One of the fundamental limitations of the methodological work to date is that the various methods have not been compared with respect to their different response shift operationalizations, corresponding interpretation of results and alternative explanations.

The current work is part of an international, interdisciplinary collaboration (see Appendix for the contributing members) to synthesize extant research on response shift $[8,25$, 26].The primary objective of the current paper is to provide a critical examination of response shift methods. We compare their implied definitions, how response shift is operationalized in terms of the type of response shift they aim to detect and whether they can adjust for and explain detected response shift, their underlying assumptions, and alternative explanations of response shift results. In so doing, we additionally propose further development of new methods that address some of the limitations of existing response shift methods and are expected to advance the field.

\section{Overview of response shift detection methods}

\section{Inventory of methods}

Based on earlier overviews [27, 28] and previous reviews, $[11,12]$ of response shift methods, we identified 11 methods. These include the then-test and appraisal method, socalled design methods [3, 5, 29], semi-structured interview as an example of a qualitative method [30], the Patient Generated Index (PGI) [31], and the Schedule for the Evaluation of Individual Quality of Life (SEIQoL) [32] to illustrate individualized methods, where we will further focus on the SEIQoL, and vignette studies to exemplify preference-based methods [33].

The remaining methods rely on different statistical methods. These are identified based on the previous scoping review [11] and an expository review [12]. The most recent scoping review was based on the search of seven library databases (Biomed, CINAHL, EMBASE, Medline, ProQuest, PsycINFO, and Web of Science) for the use of the terms "response shift," "response shift effects," "longitudinal measurement invariance," or "retrospective bias" and was limited to articles written in English and published or accepted for publication up to December 31, 2016. Some of the statistical methods, based on the framework of latent variables that are not directly observable but rather inferred (through latent variable models) from other variables that are observed (or directly measured), include Structural 
Equation Modeling (SEM) [34], Item Response Theory (IRT) [16], and Rasch Measurement Theory (RMT) [24]. Other statistical frameworks encompass Relative Importance Analysis [15], Classification and Regression Tree (CART) [14], Random Forest Regression [35], and Mixed Models and Growth Mixture Models [13] (Tables 1 and 2).

Other response shift methods have been proposed, including adaptations of the Repertory Grid Technique, Extended Q-TWiST method, Ideal Scale method, and idiographic assessment of personal goals [27]. However, these methods, requiring specific measures, have rarely been used and tested, and are therefore not included in this critical examination.

\section{Description}

All methods have requirements regarding study design. They all demand a longitudinal design with at least two or three measurement occasions, with the exception of qualitative methods that may ask the respondent to compare the present in relation to a past reference period. The then-test is the only method needing an additional measurement in addition to the baseline and follow-up PROM; other methods require the use of specific instruments (i.e., appraisal, SEIQoL, vignettes). Some statistical methods require a priori identification of subgroups (Relative Importance Analysis) or clinical explanatory variables (CART and Random Forest Regression) (Table 1 and Supplementary Table 1).

Response shift can be investigated at the individual or at the group level (i.e., by aggregating scores). Some methods can accommodate both group- and individual-level analyses (individualized methods such as the SEIQoL that allow aggregation of individual-level data), while others are used for individual-level analysis (i.e., qualitative methods) or group- or subgroup-level analysis (e.g., then-test, Vignettes, SEM) (Table 1).

\section{Definitions}

We chose the response shift working definition provided by Sprangers and Schwartz [4] and the definition proposed by Oort et al. $[6,7]$ to describe the methods. The former encompasses the definitions proposed by Golembiewski et al. [2] and Howard et al. [3], while Oort et al. adopted a different perspective, which is based on the discrepancy between observed change and target change. These definitions can be applied to all methods, whereas the Rapkin and Schwartz [5] definition requires assessing appraisal processes.

All methods operationalize the working definition of response shift, albeit indirectly, i.e., by inferring it from data. Six methods distinguish between observed change and target change, i.e., then-test, appraisal, qualitative methods, SEM,
IRT/RMT, and Mixed Models and Growth Mixture Models. However, the discrepancy between observed change and target change is operationalized differently. To exemplify, qualitative methods use the discrepancy between reported change over time and modifications of that change when asked for a reflection SEM and IRT/RMT use the discrepancy between observed change scores and change in unobserved latent variables. The other methods do not distinguish between observed change and target change (Table 1).

\section{Operationalization}

Clearly, all methods aim to detect response shift, but some methods can only detect one type of response shift. The then-test and RMT aim to assess recalibration, whereas vignettes, and Relative Importance Analysis and Mixed Models and Growth Mixture Models can assess reprioritization. IRT, CART, and Random Forest Regression aim to assess both recalibration and reprioritization, the SEIQoL is targeted at assessing reprioritization and reconceptualization, and qualitative methods and SEM aim to assess all three types of response shift. Only the appraisal method does not distinguish among type of response shift and assesses an overall response shift effect (Table 1).

Some methods can adjust for response shift, i.e., assess change while controlling for response shift. These include the then-test, SEM, IRT/RMT, Mixed Models and Growth Mixture Models. All methods, with the exception of appraisal, can accommodate exogenous variables (e.g., sociodemographic, clinical, and psychosocial variables) aimed to explain potential causes of the detected response shift effects, by for example including these variables as covariates in statistical models (Table 1).

Assumptions Some methods require reflection from the respondents on their Health-Related Quality of Life (HRQoL), functioning, and/or response behavior (i.e., appraisal, qualitative interviews), and/or life domains that are important to them and their relative importance (i.e., individualized methods such as the SEIQoL) (Table 2). Qualitative methods additionally require that respondents are able to verbalize such reflections and, dependent on the question, may be assumed to be aware of possible response shifts. The preference-based methods based on vignettes assume that ratings of vignettes can be translated into respondents' assessments of their own health state. The ability to recall is an important assumption for the use and interpretation of a number of methods, including the thentest (previous level of functioning), appraisal (previous completion of questionnaire items), qualitative methods (a past reference period), and individualized methods such as the SEIQoL (previous domains or weights). 


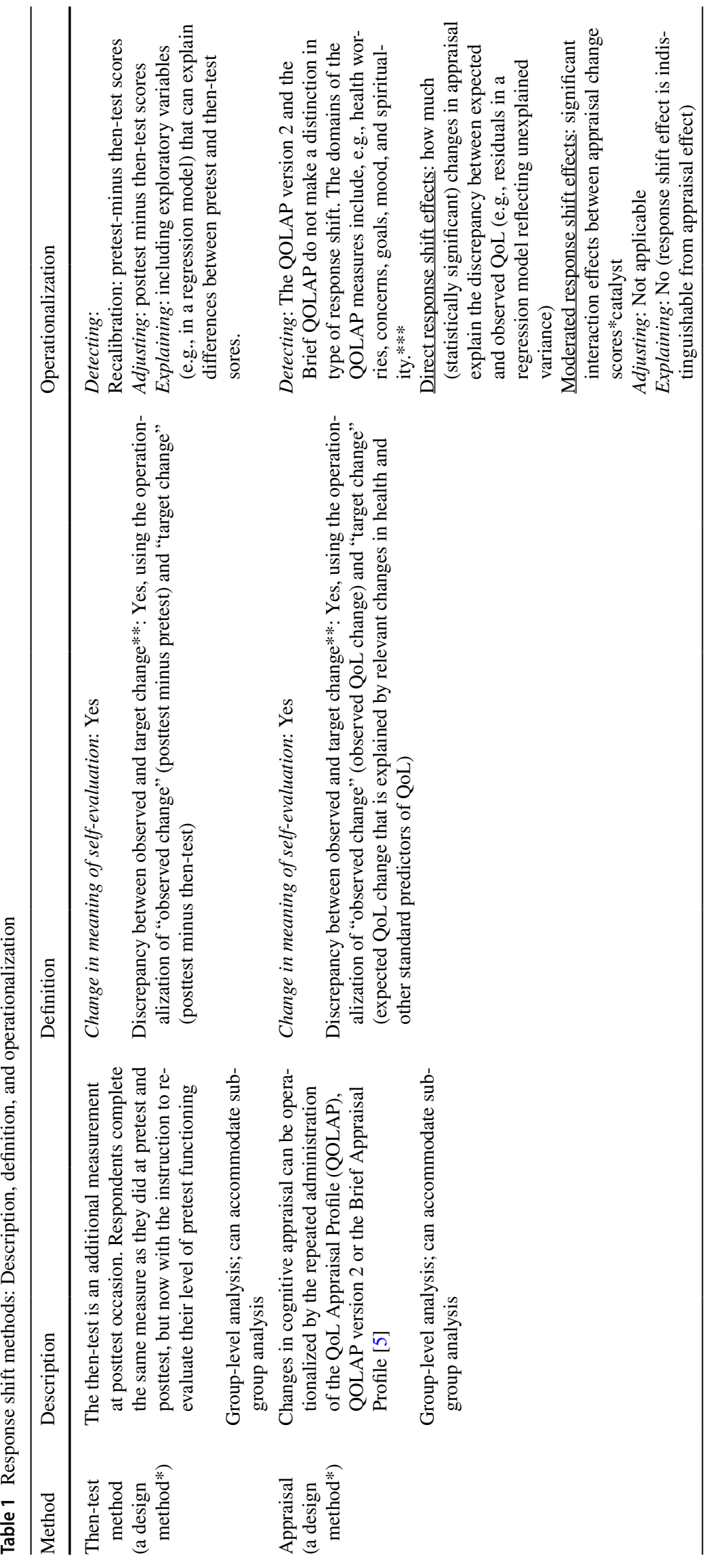




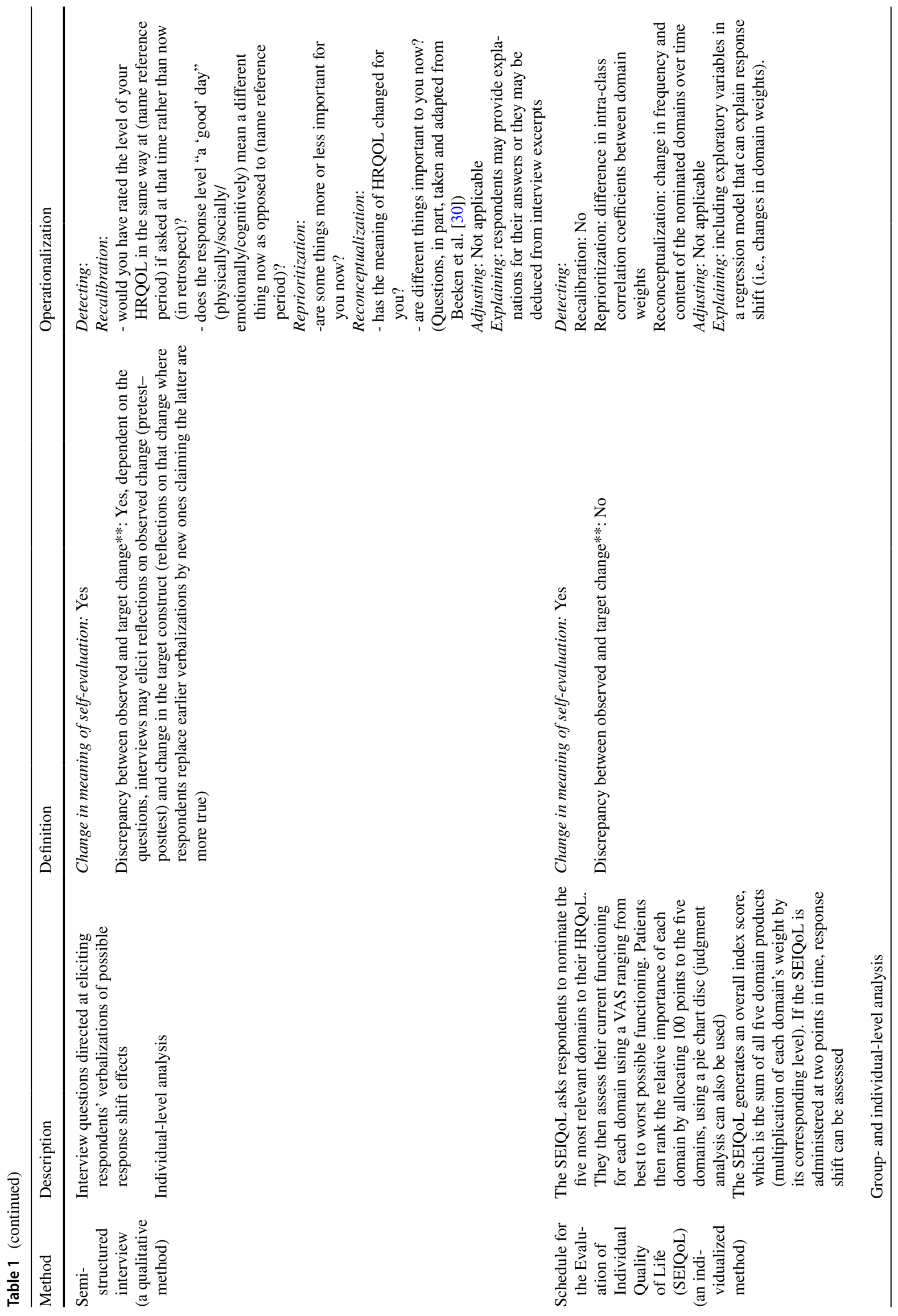




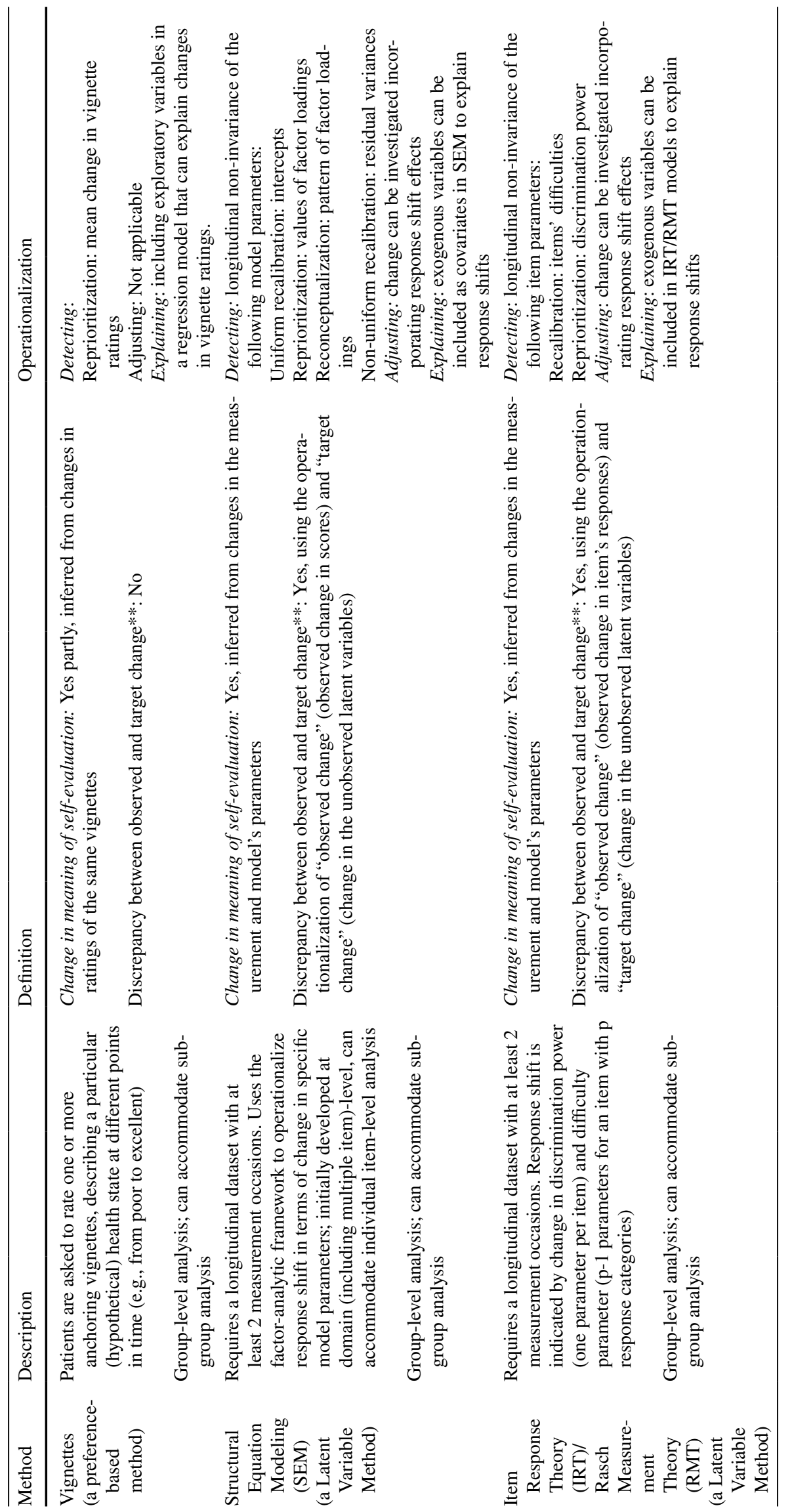




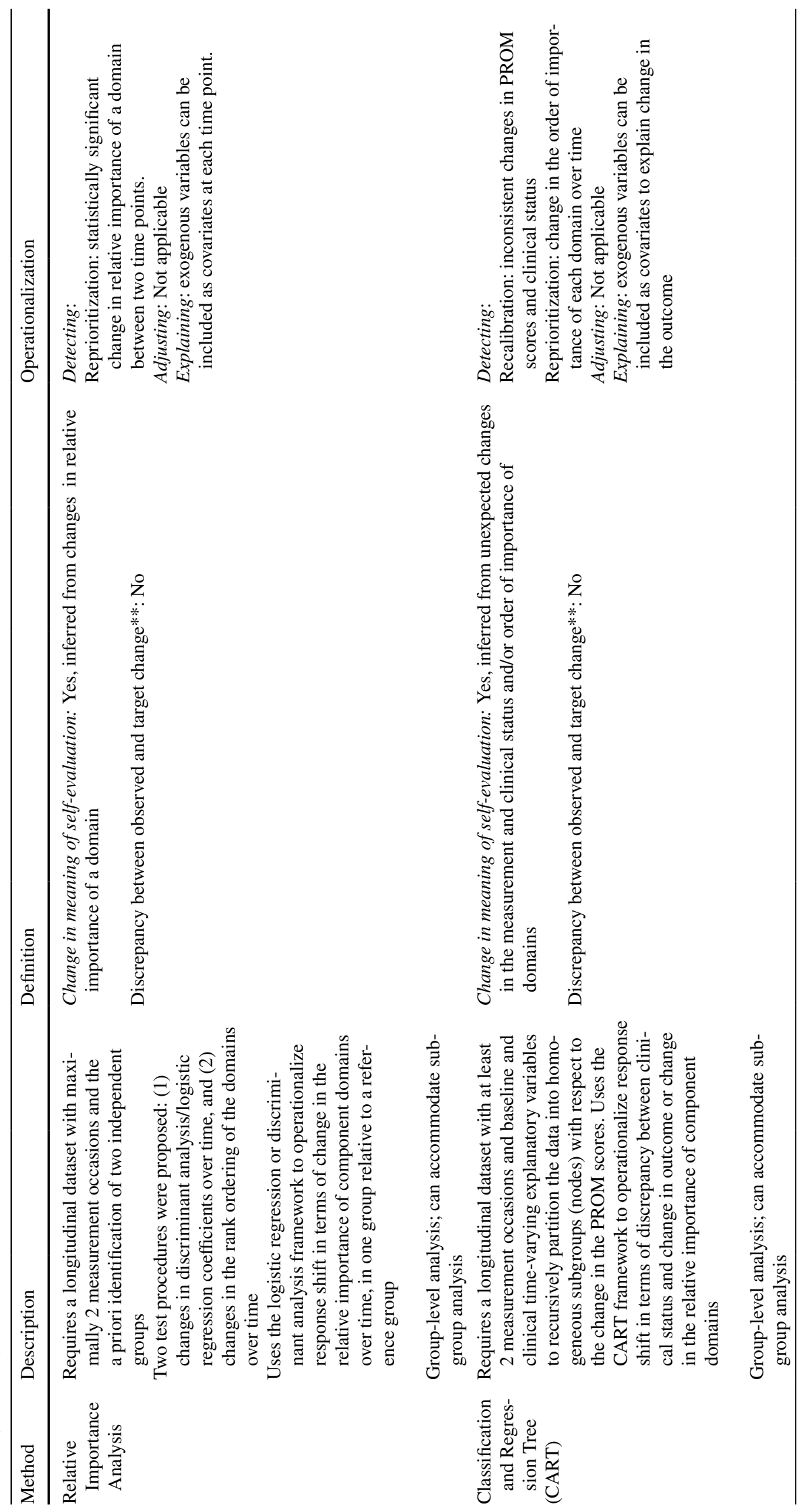




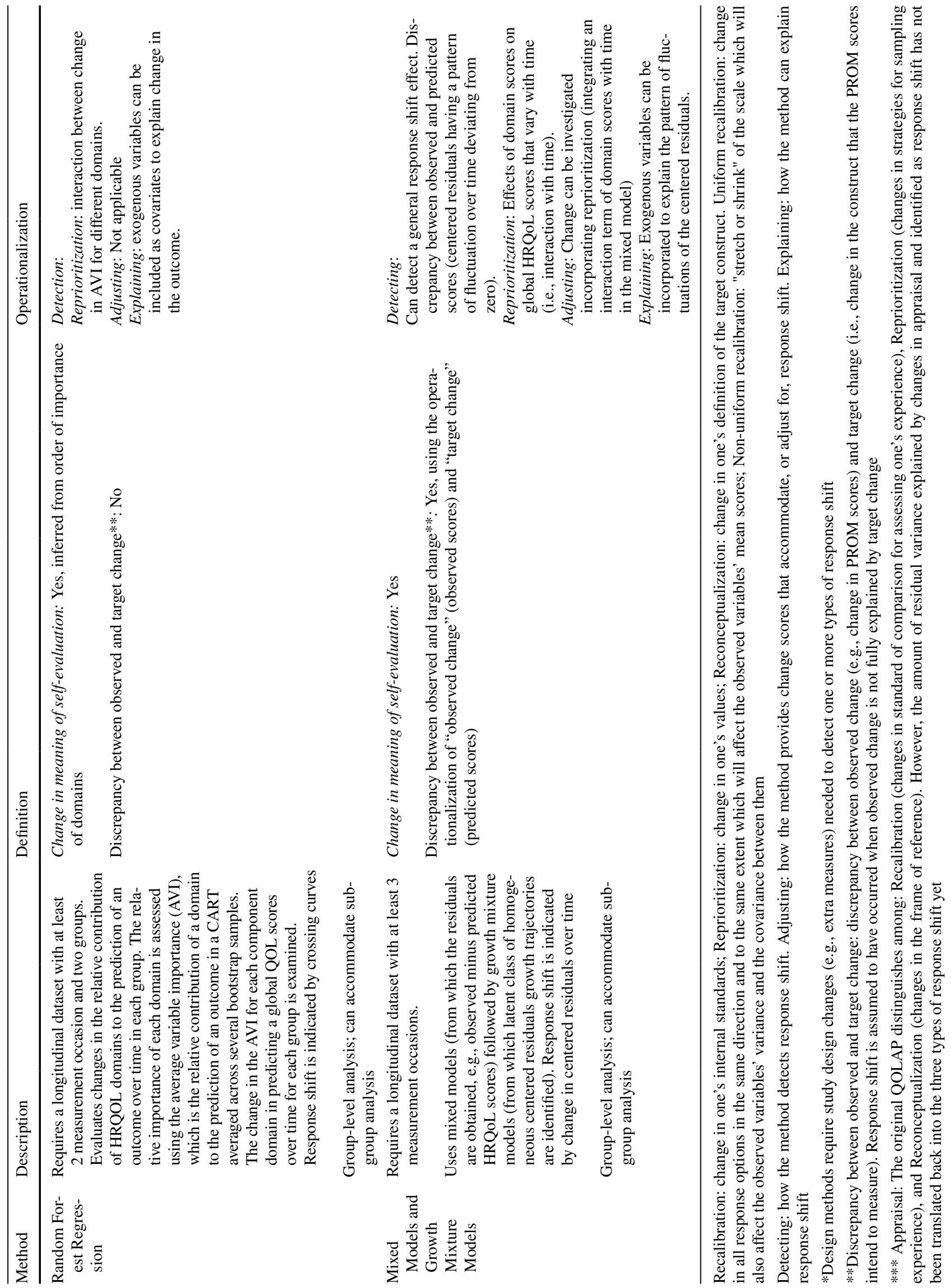




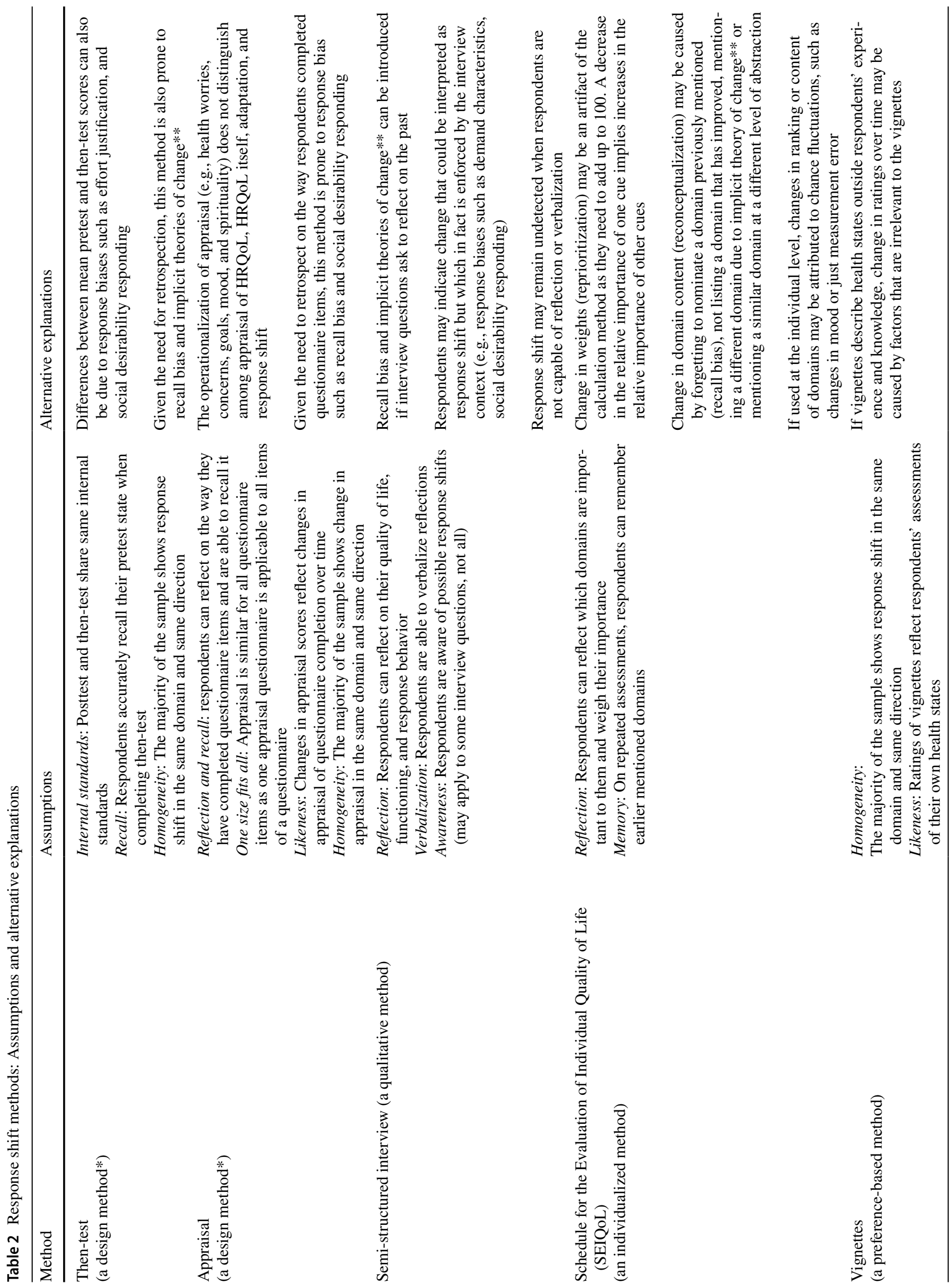




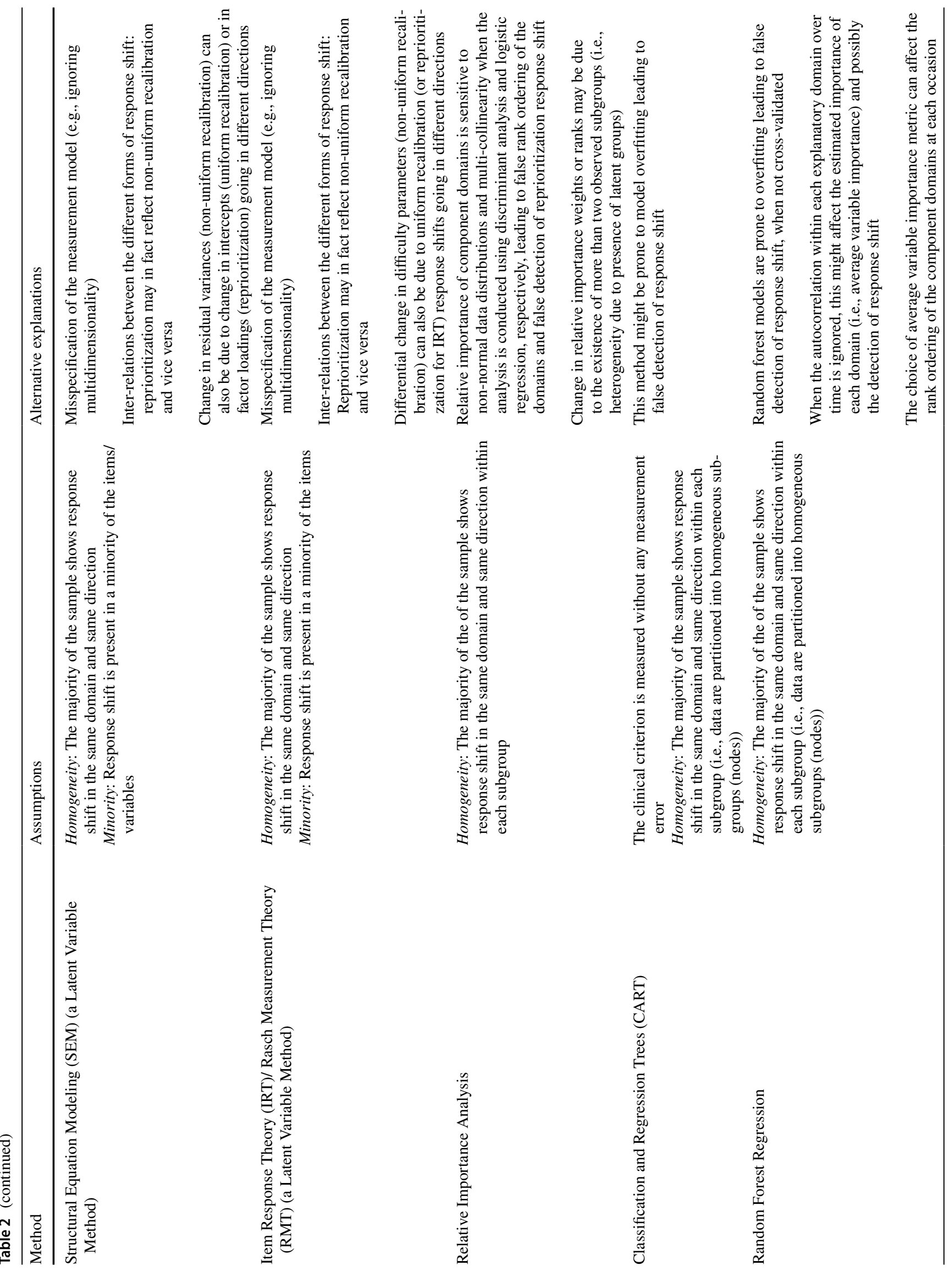


The methods that can only detect response shift at group or subgroup level (i.e., then-test, appraisal, vignettes, SEM, IRT/RMT, Relative importance analysis, CART, Random Forest Regression, and Mixed Models and Growth Mixture Models) assume that response shift is more or less homogeneous within a (sub)sample and affects the majority of the respondents in the same domain (or items) and in the same direction. SEM and IRT/RMT additionally assume that response shift is only present in a minority of the items/ variables (Table 2).

\section{Alternative explanations}

All methods are prone to the possibility of alternative explanations of the response shift results, albeit different ones. Many influences can lead to the conclusion that response shift is present when it in fact is not, or vice versa, including recall bias (i.e., then-test, appraisal, qualitative interviews, and individualized methods such as the SEIQoL), response bias such as social desirability responding (i.e., then-test, appraisal, qualitative methods), the incapacity of verbalizing one's own experience and feelings (i.e., qualitative methods), and more generally irrelevant "stimuli" for the patients (e.g., interview questions, and health state descriptions in the vignettes).

For all statistical methods, misspecification of the (measurement) model can lead to the conclusion that response shift has occurred when it has not, or vice versa. For SEM and IRT/RMT methods, inter-relations between different types of response shift may also lead to falsely concluding that one type of response shift has occurred (e.g., reprioritization) while in fact another type has (e.g., recalibration) (Table 2).

\section{Future research}

This review reveals limitations of the current response shift methods. We address the need to cautiously interpret the results of the response shift methods. Since response shift is not likely to occur in the entire group under study with the same type, magnitude, and/or direction, we suggest advancing new methods for handling inter-individual variation in change over time. Moreover, the course of response shift is often unknown and may not exclusively occur within two measurement occasions. We suggest new methods that can accommodate multiple time points.

\section{Interpreting results as response shift}

When response shift can be inferred by one of the methods (Tables 1 and 2), extra steps need to be taken to exclude alternative explanations or make these less plausible, to 
correctly interpret the results as response shift as defined by Sprangers and Schwartz [4]. For example, for the methods testing the discrepancy between observed and target change $[6,7]$, there is particular confusion about how to interpret detected response shift effects. This operationalization may indeed be a necessary but not sufficient condition for response shift to occur allowing alternative explanations for this discrepancy. For example, imagine that the item relating to the assessment of the most acute pain is one of the indicators that measure pain severity. Suppose that patients rated their acute pain to be lower at follow-up, even though their general pain severity did not change or changed to a lesser degree. This result indicates a discrepancy between observed change and target change. A possible explanation for this result may be that patients adapted to the experience of pain. It may also be that patients received pain killers that only reduced acute pain. One could argue that only the first interpretation coincides with the working definition of response shift as a change in the meaning of one's selfevaluation of a target construct as a result of recalibration [4]. Hence, examination of possible alternative explanations of the findings need to be considered and ruled out before the conclusion that response shift has occurred is warranted.

\section{Inter-individual variation}

New insights about adaptations to health events could be gained through exploration of heterogeneity in response shift, where different people experience different types and/or directions of response shift, or no response shift. Such insights could for example be used to develop new treatments and interventions for patients. However, most response shift methods typically assume response shift to be homogeneous, ignoring that interpretation of items may be influenced by cultural, developmental, or personality differences, contextual factors or life circumstances, and/or because of different health experiences or events.

A number of methods have been forwarded to aid the identification of variation in response shift between individuals (Table 3 ). For example, inter-individual variation, based on residuals, was examined using Mixed Models and Growth Mixture Models [13]. Among some challenges encountered with this method, alternative explanations, other than response shift, for the discrepancies in the direction of the residuals were cited by the authors. Other studies have explicitly investigated the effect of measured covariates on response shift in longitudinal data. For example, KingKallimanis et al. [36] demonstrated the use of SEM with age, sex, and CD4-cell counts as covariates, to explain variation in response shift on physical and mental HRQoL of HIV/AIDS patients. The authors however noted some challenges which included fitting multiple models to the data and sample size requirements. Lix et al. [37] approached inter-individual variation by stratifying their SEM analysis, according to disease activity, an a priori known source of heterogeneity. The authors found evidence of different types of response shift in these different groups and noted that this approach is useful when only a small number of measured covariates are investigated. Salmon et al. [22] proposed using a combination of Mixed Models and Growth Mixture Models and SEM to detect response shift among a cohort of breast cancer patients who provided self-reported cancerrelated fatigue. Latent classes that showed different types of response shift were identified. The authors however noted some concerns, including mixed model misspecifications [38] and ignoring the uncertainty of classification for the latent classes [39, 40].

Clearly, new tools are needed to explore inter-individual variation in response shift especially when the sources of variation are not known a priori. Latent Variable Mixture Models (LVMMs) have been put forward for this purpose [41]. Sawatzky et al. [42] proposed the use of LVMMs models to identify homogenous samples with respect to a unidimensional measurement model, and described the implications and sources of sample heterogeneity. However, challenges to extend these models for response shift analysis remain [12] and further research is needed to examine how well LVMMs perform in longitudinal data and to test implications of various specifications (e.g., whether to correlate item residual variances over time) and secure computational resources [43, 44].

\section{Multiple time points}

Response shift analysis is particularly relevant after the occurrence of a significant health event (catalyst; [4]). Hence, many studies have employed two measurement occasions: before and after such an event (e.g., a diagnosis, a treatment). Whereas such an approach may be justified if one wishes to study the effect of a clearly identified health event, limiting response shift detection to only two measurement occasions may be too restrictive in other instances. For example, some health state changes may have gradual shifts either or not in combination with sudden shifts, such as in recovery processes. Response shift could also be the result of the mere passage of time [4]. In these instances, the use of multiple time points may facilitate the detection of response shift effects.

Response shift detection methods that can accommodate multiple time points are scarce but some have been suggested (Table 4). Oort's [34] longitudinal SEM approach was extended to four time points and a clinical application in stroke patients was provided [45]. Some challenges related to sample size were however noted.

Another extension of Oort's [34] SEM approach is the longitudinal three-mode SEM model with Kronecker 


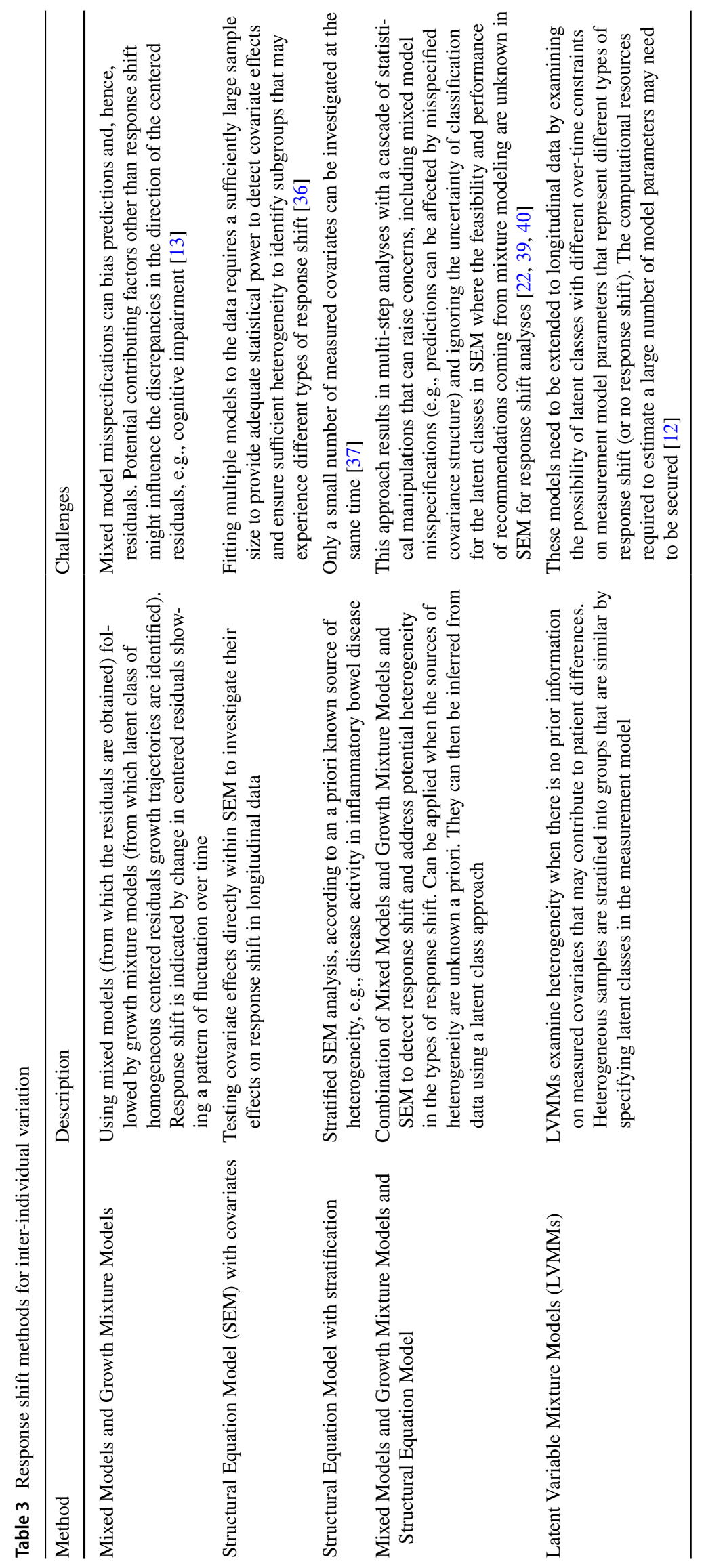







product restrictions $[46,47]$ which can accommodate many measurement occasions. Some challenges have nevertheless been put forward by the authors including the evaluation of model fit.

Mixed models can also accommodate multiple measurement occasions to explore reprioritization response shift [48]. This approach can however be impacted by strong correlations among predictor variables but there are solutions to overcome such multi-collinearity which can be implemented [49].

Methods particularly suited for item-level analysis, such as IRT and RMT [16, 24, 50], can also be extended to accommodate multiple time points. However, this may lead to models with numerous parameters to estimate and to model specification challenges in modeling item dependencies over time $[24,50]$. Alternative modeling strategies are needed.

Bayesian joint growth models [51] may be used, which require specification of proper prior distributions. Currently, such an approach or [52] a frequentist multiple time points framework not requiring prior knowledge is being explored. Irrespective of the chosen framework, interpreting and translating the trajectories of the item parameters to the working response shift definition might be a challenge. Moreover, methods for identifying key time points where we could consider that a clinically meaningful change in items' parameters has occurred might also be needed. Joint models with change of the response shift parameters [53] that allow handling interval censoring could be promising since the occurrence of response shift is assumed to lie within an interval without being observed exactly [54].

\section{Epilogue}

Despite operational differences among the eleven response shift methods, they share a common working definition related to a change in the meaning of one's self-evaluation of a target construct [4]. Not surprisingly, this critical examination showed that no single response shift method is optimal in all situations; each has its strengths and limitations. Therefore, it is too early in the development of the field of response shift research to provide a priori recommendations regarding which method to use in a certain situation. The key finding is that response shift results cannot be accepted at face value and extra steps need to be taken to correctly interpret the results. This calls for training in the application of response shift methods and carefully designed studies. First, a rationale for the occurrence of response shift is needed that is based on substantive theory and/or clinical knowledge. Second, a precise objective needs to be formulated detailing the problem that will be examined. For example, will response shift be addressed as measurement bias that needs to be adjusted or as an interesting phenomenon in itself, shedding light on change per se. Third, wherever possible, hypotheses need to be formulated a priori about which type of response shift is expected, in which direction, in which domain, within which time period(s), and for which subsample. Fourth, methods need to be selected that correspond with the study objective and the characteristics of the data that will be collected. For example, whether the aim is to investigate domain-level, item-level response shift, or both will guide the selection of the method. Fifth, alternative explanations need to be identified for which the current overview per method is expected to be helpful. However, they can also be debated and it may be difficult to disentangle response shift from alternative explanations. For example, dimensionality and heterogeneity can also explain the discrepancy between observed and target change. Sixth, studies must be designed so that it is possible to rule out alternative explanations, or make them less plausible. This includes incorporating a control or comparison group into the design and external criterion measures of change (e.g., self-reported transition scores). We also recommend adopting the response shift method that is most appropriate for a given study (e.g., statistical methods versus individualized methods). If possible, a qualitative method may be added to investigate the causes of response shift or to provide supporting evidence [27]. However, these methods may not be suited for triangulation of group-level analytical methods, given their individual-level analytical perspective. Finally, response shift is frequently examined through secondary analysis of existing data using statistical methods. Careful attention should be given to the optimal implementation of such analyses. We refer the reader to the guidelines proposed by Schwartz et al. [9].

The current critical examination also highlighted the need to further develop new methods to investigate inter-individual variation in change over time and across multiple time points. We presented a range of promising techniques. It should be noted that while these new methods may be useful and help to advance the field of response shift research, they are not likely a panacea as they also need to be scrutinized for their susceptibility to alternative explanations. An additional way forward is to apply different methods to the same dataset to see how the methods compare with respect to detecting the same type of response shift. While this seems to be a valuable approach, it has two main caveats. First, because we do not know the "truth" (i.e., whether response shift is present or absent in the population), the findings will not tell us whether the different methods are sensitive to detect response shift. Second, different methods require different data. In a head-to-head comparison of three statistical approaches using a single dataset of patients with multiple sclerosis, it was necessary to accommodate the data to meet the requirements of each statistical approach, 
thereby hampering direct comparisons [21]. Perhaps a more promising path forward involves the conduct of simulation studies, which can provide insight into the performance of the extant and future methods, given known response shift effects and based on requisite simulated data that enable the comparison of the methods [55] (e.g., adequate control of Type 1 error, sufficient statistical power, examining bias). Importantly, such studies would be able to examine the effects of falsification [25] by generating simulated data that are inconsistent with response shift and examining the extent to which the methods are able to rule out alternative explanations. The use of simulation studies would also allow the assessment and the comparison of the robustness of different methods to certain model assumptions (e.g., normal distribution) and/or missing data, particularly missing not at random (MNAR) data which are likely in longitudinal studies with patients [56].

It is our hope that this critical examination will stimulate research on the further advancement of response shift research by carefully applying extant and novel methods, cautiously interpreting their results, and conducting simulation studies of extant and novel methods.

Supplementary Information The online version of this article (https:// doi.org/10.1007/s11136-020-02755-4) contains supplementary material, which is available to authorized users.

Funding Department of Medical Psychology, Amsterdam University Medical Centers, the Netherlands and SPHERE, Université de Nantes, Université de Tours, France.

\section{Compliance with ethical standards}

Conflict of interest All authors declared that they have no competing interest.

Open Access This article is licensed under a Creative Commons Attribution 4.0 International License, which permits use, sharing, adaptation, distribution and reproduction in any medium or format, as long as you give appropriate credit to the original author(s) and the source, provide a link to the Creative Commons licence, and indicate if changes were made. The images or other third party material in this article are included in the article's Creative Commons licence, unless indicated otherwise in a credit line to the material. If material is not included in the article's Creative Commons licence and your intended use is not permitted by statutory regulation or exceeds the permitted use, you will need to obtain permission directly from the copyright holder. To view a copy of this licence, visit http://creativecommons.org/licenses/by/4.0/.

\section{References}

1. Schwartz, C. E., \& Rapkin, B. D. (2004). Reconsidering the psychometrics of quality of life assessment in light of response shift and appraisal. Health and Quality of Life Outcomes, 2(1), 16. https ://doi.org/10.1186/1477-7525-2-16.

2. Golembiewski, R. T. (1976). Measuring change and persistence in human affairs: Types of change generated by OD designs. The
Journal of Applied Behavioral Science, 12(2), 133-157. https:// doi.org/10.1177/002188637601200201.

3. Howard, G. S., Ralph, K. M., Gulanick, N. A., Maxwell, S. E., Nance, D. W., \& Gerber, S. K. (1979). Internal invalidity in pretest-posttest self-report evaluations and a re-evaluation of retrospective pretests. Applied Psychological Measurement, 3(1), 1-23. https://doi.org/10.1177/014662167900300101.

4. Sprangers, M. A., \& Schwartz, C. E. (1999). Integrating response shift into health-related quality of life research: a theoretical model. Social Science \& Medicine, 48(11), 1507-1515.

5. Rapkin, B. D., \& Schwartz, C. E. (2004). Toward a theoretical model of quality-of-life appraisal: Implications of findings from studies of response shift. Health and Quality of Life Outcomes, 2, 14. https://doi.org/10.1186/1477-7525-2-14.

6. Oort, F. J. (2005). Towards a formal definition of response shift (in reply to G.W. Donaldson). Quality of Life Research An International Journal of Quality of Life Aspects of Treatment, Care and Rehabilitation, 14(10), 2353-2355. https://doi.org/10.1007/s1113 6-005-3978-1.

7. Oort, F. J., Visser, M. R. M., \& Sprangers, M. A. G. (2009). Formal definitions of measurement bias and explanation bias clarify measurement and conceptual perspectives on response shift. Journal of Clinical Epidemiology, 62(11), 1126-1137. https:// doi.org/10.1016/j.jclinepi.2009.03.013.

8. Vanier, A., Oort, F. J., McClimans, L., Ow, N., Gulek, B. G., Böhnke, J. R., ... \& the Response Shift - in Sync Working Group. (n.d.). Response shift in patient-reported outcomes measures: A formal definition and a revised model., Manuscript under review (same issue).

9. Schwartz, C. E., Ahmed, S., Sawatzky, R., Sajobi, T., Mayo, N., Finkelstein, J., ..., \& Sprangers, M. A. G. (2013). Guidelines for secondary analysis in search of response shift. Quality of Life Research: An International Journal of Quality of Life Aspects of Treatment, Care and Rehabilitation, 22(10), 2663-2673. https:// doi.org/10.1007/s11136-013-0402-0.

10. Schwartz, C. E., \& Sprangers, M. A. G. (2010). Guidelines for improving the stringency of response shift research using the thentest. Quality of Life Research: An International Journal of Quality of Life Aspects of Treatment, Care and Rehabilitation, 19(4), 455-464. https://doi.org/10.1007/s11136-010-9585-9.

11. Sajobi, T. T., Brahmbatt, R., Lix, L. M., Zumbo, B. D., \& Sawatzky, R. (2018). Scoping review of response shift methods: Current reporting practices and recommendations. Quality of Life Research: An International Journal of Quality of Life Aspects of Treatment, Care and Rehabilitation, 27(5), 1133-1146. https:// doi.org/10.1007/s11136-017-1751-x.

12. Sawatzky, R., Sajobi, T. T., Brahmbhatt, R., Chan, E. K. H., Lix, L. M., \& Zumbo, B. D. (2017). Longitudinal change in response processes: A response shift perspective. In B. D. Zumbo \& A. M. Hubley (Eds.), Understanding and investigating response processes in validation research (pp. 251-276). Cham: Springer.

13. Mayo, N. E., Scott, S. C., Dendukuri, N., Ahmed, S., \& WoodDauphinee, S. (2008). Identifying response shift statistically at the individual level. Quality of Life Research: An International Journal of Quality of Life Aspects of Treatment, Care and Rehabilitation, 17(4), 627-639. https://doi.org/10.1007/s11136-008-9329-2.

14. Li, Y., \& Rapkin, B. (2009). Classification and regression tree uncovered hierarchy of psychosocial determinants underlying quality-of-life response shift in HIV/AIDS. Journal of Clinical Epidemiology, 62(11), 1138-1147. https://doi.org/10.1016/j.jclin epi.2009.03.021.

15. Lix, L. M., Sajobi, T. T., Sawatzky, R., Liu, J., Mayo, N. E., Huang, Y., ..., \& Bernstein, C. N. (2013). Relative importance measures for reprioritization response shift. Quality of Life Research: An International Journal of Quality of Life Aspects of 
Treatment, Care and Rehabilitation, 22(4), 695-703. https://doi. org/10.1007/s11136-012-0198-3.

16. Guilleux, A., Blanchin, M., Vanier, A., Guillemin, F., Falissard, B., Schwartz, C. E., ..., \& Sébille, V. (2015). RespOnse Shift ALgorithm in Item response theory (ROSALI) for response shift detection with missing data in longitudinal patient-reported outcome studies. Quality of Life Research: An International Journal of Quality of Life Aspects of Treatment, Care and Rehabilitation, 24(3), 553-564. https://doi.org/10.1007/s11136-014-0876-4.

17. Gerlich, C., Schuler, M., Jelitte, M., Neuderth, S., Flentje, M., Graefen, M., ..., \& Faller, H. (2016). Prostate cancer patients' quality of life assessments across the primary treatment trajectory: "True" change or response shift? Acta Oncologica (Stockholm, Sweden), 55(7), 814-820. https://doi.org/10.3109/02841 86X.2015.1136749.

18. Reissmann, D. R., Erler, A., Hirsch, C., Sierwald, I., Machuca, C., \& Schierz, O. (2018). Bias in retrospective assessment of perceived dental treatment effects when using the Oral Health Impact Profile. Quality of Life Research: An International Journal of Quality of Life Aspects of Treatment, Care and Rehabilitation, 27(3), 775-782. https://doi.org/10.1007/s11136-017-1725-z.

19. Visser, M. R. M., Oort, F. J., \& Sprangers, M. A. G. (2005). Methods to detect response shift in quality of life data: A convergent validity study. Quality of Life Research: An International Journal of Quality of Life Aspects of Treatment, Care and Rehabilitation, 14(3), 629-639. https://doi.org/10.1007/s11136-004-2577-x.

20. Ahmed, S., Mayo, N. E., Wood-Dauphinee, S., Hanley, J. A., \& Cohen, S. R. (2005). The structural equation modeling technique did not show a response shift, contrary to the results of the then test and the individualized approaches. Journal of Clinical Epidemiology, 58(11), 1125-1133. https://doi.org/10.1016/j.jclin epi.2005.03.003.

21. Schwartz, C. E., Sprangers, M. A. G., Oort, F. J., Ahmed, S., Bode, R., Li, Y., \& Vollmer, T. (2011). Response shift in patients with multiple sclerosis: An application of three statistical techniques. Quality of Life Research: An International Journal of Quality of Life Aspects of Treatment, Care and Rehabilitation, 20(10), 1561-1572. https://doi.org/10.1007/s11136-011-0056-8.

22. Salmon, M., Blanchin, M., Rotonda, C., Guillemin, F., \& Sébille, V. (2017). Identifying patterns of adaptation in breast cancer patients with cancer-related fatigue using response shift analyses at subgroup level. Cancer Medicine, 6(11), 2562-2575. https:// doi.org/10.1002/cam4.1219.

23. Preiß, M., Friedrich, M., Stolzenburg, J.-U., Zenger, M., \& Hinz, A. (2019). Response shift effects in the assessment of urologic cancer patients' quality of life. European Journal of Cancer Care, 28(4), e13027. https://doi.org/10.1111/ecc.13027.

24. Blanchin, M., Guilleux, A., Hardouin, J.-B., \& Sébille, V. (2020). Comparison of structural equation modelling, item response theory and Rasch measurement theory-based methods for response shift detection at item level: A simulation study. Statistical Methods in Medical Research, 29(4), 1015-1029. https://doi. org/10.1177/0962280219884574.

25. Sprangers, M. A. G., Sajobi, Tolulope T., Vanier, A., Mayo, N. E., Sawatzky, R., Lix, L. M., et al. \& The Response Shift-in Sync Working Group. (2021). Response shift in results of patientreported outcome measures: A commentary to the response shift - in sync working group initiative. Quality of Life Research. https ://doi.org/10.1007/s11136-020-02747-4.

26. Sawatzky, R., Kwon, J.-Y., Barclay, R., Chauhan, C., Frank, L., van den Hout, W., et al. \& The Response Shift—in Sync Working Group (2021). Implications of response shift for micro-, meso-, and macro-level healthcare decision-making using results of patient-reported outcome measures. Quality of Life Research,. https://doi.org/10.1007/s11136-021-02766-9.
27. Schwartz, C. E., \& Sprangers, M. A. G. (1999). Methodological approaches for assessing response shift in longitudinal healthrelated quality-of-life research. Social Science \& Medicine, 48(11), 1531-1548.

28. Barclay-Goddard, R., Epstein, J. D., \& Mayo, N. E. (2009). Response shift: A brief overview and proposed research priorities. Quality of Life Research: An International Journal of Quality of Life Aspects of Treatment, Care and Rehabilitation, 18(3), 335-346. https://doi.org/10.1007/s11136-009-9450-x.

29. Sprangers, M. A., Van Dam, F. S., Broersen, J., Lodder, L., Wever, L., Visser, M. R., ..., \& Smets, E. M. (1999). Revealing response shift in longitudinal research on fatigue-the use of the thentest approach. Acta Oncologica (Stockholm, Sweden), 38(6), 709-718.

30. Beeken, R. J., Eiser, C., \& Dalley, C. (2011). Health-related quality of life in haematopoietic stem cell transplant survivors: A qualitative study on the role of psychosocial variables and response shifts. Quality of Life Research: An International Journal of Quality of Life Aspects of Treatment, Care and Rehabilitation, 20(2), 153-160. https://doi.org/10.1007/s1113 6-010-9737-y.

31. Ahmed, S., Mayo, N. E., Wood-Dauphinee, S., Hanley, J. A., $\&$ Cohen, S. R. (2005). Using the Patient Generated Index to evaluate response shift post-stroke. Quality of Life Research: An International Journal of Quality of Life Aspects of Treatment, Care and Rehabilitation, 14(10), 2247-2257. https://doi. org/10.1007/s11136-005-8118-4.

32. Ring, L., Höfer, S., Heuston, F., Harris, D., \& O'Boyle, C. A. (2005). Response shift masks the treatment impact on patient reported outcomes (PROs): The example of individual quality of life in edentulous patients. Health and Quality of Life Outcomes, 3, 55. https://doi.org/10.1186/1477-7525-3-55.

33. Korfage, I. J., de Koning, H. J., \& Essink-Bot, M.-L. (2007). Response shift due to diagnosis and primary treatment of localized prostate cancer: A then-test and a vignette study. Quality of Life Research, 16(10), 1627-1634. https://doi.org/10.1007/ s11136-007-9265-6.

34. Oort, F. J. (2005). Using structural equation modeling to detect response shifts and true change. Quality of Life Research: An International Journal of Quality of Life Aspects of Treatment, Care and Rehabilitation, 14(3), 587-598.

35. Boucekine, M., Boyer, L., Baumstarck, K., Millier, A., Ghattas, B., Auquier, P., \& Toumi, M. (2015). Exploring the response shift effect on the quality of life of patients with schizophrenia: An application of the random forest method. Medical Decision Making: An International Journal of the Society for Medical Decision Making, 35(3), 388-397. https://doi. org/10.1177/0272989X14559273.

36. King-Kallimanis, B. L., Oort, F. J., \& Garst, G. J. A. (2010). Using structural equation modelling to detect measurement bias and response shift in longitudinal data. AStA Advances in Statistical Analysis, 94(2), 139-156. https://doi.org/10.1007/s1018 2-010-0129-y.

37. Lix, L. M., Chan, E. K. H., Sawatzky, R., Sajobi, T. T., Liu, J., Hopman, W., \& Mayo, N. (2016). Response shift and disease activity in inflammatory bowel disease. Quality of Life Research: An International Journal of Quality of Life Aspects of Treatment, Care and Rehabilitation, 25(7), 1751-1760. https ://doi.org/10.1007/s11136-015-1188-z.

38. Jacqmin-Gadda, H., Sibillot, S., Proust, C., Molina, J.-M., \& Thiébaut, R. (2007). Robustness of the linear mixed model to misspecified error distribution. Computational Statistics \& Data Analysis, 51(10), 5142-5154. https://doi.org/10.1016/j. csda.2006.05.021.

39. Bolck, A., Croon, M., \& Hagenaars, J. (2004). Estimating latent structure models with categorical variables: One-step versus three-step estimators. Political Analysis, 12(1), 3-27. 
40. Vermunt, J. K. (2010). Latent class modeling with covariates: Two improved three-step approaches. Political Analysis, 18(4), 450-469.

41. Muthén, B. O. (2001). Latent variable mixture modeling. New developments and techniques in structural equation modeling. New York: Psychology Press.

42. Sawatzky, R., Ratner, P. A., Kopec, J. A., \& Zumbo, B. D. (2012). Latent variable mixture models: a promising approach for the validation of patient reported outcomes. Quality of Life Research: An International Journal of Quality of Life Aspects of Treatment, Care and Rehabilitation, 21(4), 637-650. https:// doi.org/10.1007/s11136-011-9976-6.

43. Lubke, G. H., \& Luningham, J. (2017). Fitting latent variable mixture models. Behaviour Research and Therapy, 98, 91-102. https://doi.org/10.1016/j.brat.2017.04.003.

44. Tueller, S., \& Lubke, G. (2010). Evaluation of structural equation mixture models parameter estimates and correct class assignment. Structural Equation Modeling: A Multidisciplinary Journal, 17(2), 165-192. https://doi.org/10.1080/10705511003659318.

45. Barclay-Goddard, R., Lix, L. M., Tate, R., Weinberg, L., \& Mayo, N. E. (2009). Response shift was identified over multiple occasions with a structural equation modeling framework. Journal of Clinical Epidemiology, 62(11), 1181-1188. https://doi. org/10.1016/j.jclinepi.2009.03.014.

46. Verdam, M. G. E., \& Oort, F. J. (2014). Measurement bias detection with Kronecker product restricted models for multivariate longitudinal data: An illustration with health-related quality of life data from thirteen measurement occasions. Frontiers in Psychology, 5, 1022. https://doi.org/10.3389/fpsyg.2014.01022.

47. Verdam, M. G. E., \& Oort, F. J. (2019). The analysis of multivariate longitudinal data: An instructive application of the longitudinal three-mode model. Multivariate Behavioral Research, 54(4), 457-474. https://doi.org/10.1080/00273171.2018.1520072.

48. Lowy, A., \& Bernhard, J. (2004). Quantitative assessment of changes in patients' constructs of quality of life: An application of multilevel models. Quality of Life Research: An International Journal of Quality of Life Aspects of Treatment, Care and Rehabilitation, 13(7), 1177-1185. https://doi.org/10.1023/ B:QURE.0000037510.17893.d2.

49. Cheng, J., Edwards, L. J., Maldonado-Molina, M. M., Komro, K. A., \& Muller, K. E. (2010). Real longitudinal data analysis for real people: Building a good enough mixed model. Statistics in Medicine, 29(4), 504-520. https://doi.org/10.1002/sim.3775.

50. Liu, Y., Millsap, R. E., West, S. G., Tein, J.-Y., Tanaka, R., \& Grimm, K. J. (2017). Testing measurement invariance in longitudinal data with ordered-categorical measures. Psychological Methods, 22(3), 486-506. https://doi.org/10.1037/met0000075.

51. Verhagen, J., \& Fox, J.-P. (2013). Longitudinal measurement in health-related surveys. A Bayesian joint growth model for multivariate ordinal responses. Statistics in Medicine, 32(17), 2988 3005. https://doi.org/10.1002/sim.5692.

52. Bishop, J., Geiser, C., \& Cole, D. A. (2015). Modeling latent growth with multiple indicators: A comparison of three approaches. Psychological Methods, 20(1), 43-62. https://doi. org/10.1037/met0000018.

53. Dantan, E., Joly, P., Dartigues, J.-F., \& Jacqmin-Gadda, H. (2011). Joint model with latent state for longitudinal and multistate data. Biostatistics (Oxford, England), 12(4), 723-736. https://doi. org/10.1093/biostatistics/kxr003.

54. Zhang, Z., \& Sun, J. (2010). Interval censoring. Statistical Methods in Medical Research, 19(1), 53-70. https://doi. org/10.1177/0962280209105023.

55. Hinds, A. M., Sajobi, T. T., Sebille, V., Sawatzky, R., \& Lix, L. M. (2018). A systematic review of the quality of reporting of simulation studies about methods for the analysis of complex longitudinal patient-reported outcomes data. Quality of Life Research: An International Journal of Quality of Life Aspects of Treatment, Care and Rehabilitation, 27(10), 2507-2516. https:// doi.org/10.1007/s11136-018-1861-0.

56. de Bock, É., Hardouin, J.-B., Blanchin, M., Le Neel, T., Kubis, G., Bonnaud-Antignac, A., ..., \& Sébille, V. (2016). Rasch-family models are more valuable than score-based approaches for analysing longitudinal patient-reported outcomes with missing data. Statistical Methods in Medical Research, 25(5), 2067-2087. https ://doi.org/10.1177/0962280213515570.

Publisher's Note Springer Nature remains neutral with regard to jurisdictional claims in published maps and institutional affiliations. 